\title{
Herz und Weaning
}

\author{
The Heart and Weaning
}

Autoren

Institute

\section{F. J. Meyer ${ }^{1}$, B. Schönhofer ${ }^{2}$}

Abt. Innere Medizin III, Kardiologie, Angiologie, Pneumologie, Universitätsklinikum, Heidelberg

2 Klinikum der Region Hannover, Krankenhaus Oststadt-Heidehaus, Abt. Pneumologie und internistische Intensivmedizin, Hannover

\section{Bibliografie}

DOI $10.1055 / \mathrm{s}-0028-1119719$

Online-Publikation: 15. 4. 2009

Pneumologie 2009; 63: 276-281

(c) Georg Thieme Verlag KG

Stuttgart · New York

ISSN 0934-8387

Korrespondenzadresse

Priv.-Doz. Dr. med.

F. Joachim Meyer

Abt. Innere Medizin III

Universitätsklinikum

Im Neuenheimer Feld 410

69120 Heidelberg

Joachim.Meyer@med.uni-

heidelberg.de

Serienherausgeber

S. Rosseau, Berlin

B. Schönhofer, Hannover

\section{Zusammenfassung}

$\checkmark$

Der Beginn einer maschinellen Beatmung und die Rückkehr zur Spontanatmung (Weaning) induzieren erhebliche Änderungen des intrathorakalen Drucks mit Konsequenzen für die Herz-KreislaufFunktion. Trotz dieser bekannten pathophysiologischen Zusammenhänge wird die kardiovaskuläre Situation der Patienten häufig vor und beim Weaning nicht ausreichend beachtet. Die publizierten und in Leitlinien empfohlenen Kriterien für Beginn des Weanings, Extubation und Reintubation sind notgedrungen allgemein gehalten. Die Erkenntnis, dass eine kardiale Dysfunktion ein erfolgreiches Weaning verhindern kann, basiert auf Studien bei Patienten mit bekannter kardiovaskulärer Erkrankung. Tatsächlich haben viele Intensivpatienten eine latente oder noch nicht diagnostizierte kardiale Dysfunktion. Zudem limitiert die intensivmedizinische Grunderkrankung häufig die kardiovaskulären Kompensationsoptionen. Daher scheitert das Weaning bei nicht wenigen Intensivpatienten an einer ungenügenden hämodynamischen Reserve. Diese Arbeit stellt die klinisch relevanten Grundlagen für das erfolgreiche Weaning mit besonderer Berücksichtigung der kardiovaskulären Situation des Patienten dar.

\section{Abstract \\ $\nabla$}

The initiation of and weaning from mechanical ventilation with extubation cause significant changes in intrathoracic pressures and have profound consequences for the cardiovascular function. However, in spite of the known pathophysiological relationships, frequently little attention is paid to the cardiovascular situation during the weaning period. The currently available guidelines concerning weaning and extubation/reintubation are based on only limited evidence and are thus rather general. Moreover, recommendations for the cardiovascular management during weaning reflect mainly the pathophysiological rationale. In patients with known cardiac disease, studies have demonstrated the necessity for a prolonged weaning process. On the contrary, many intensive care patients have subclinical or undiagnosed cardiac disease, and cardiovascular compensation mechanisms are frequently hampered by the underlying critical illness. Therefore, in a significant proportion of patients, weaning and extubation attempts fail due to the patient's limited cardiovascular reserves. This review summarises the relevant information for a successful weaning and extubation with special respect to the cardiovascular function.

\section{Einleitung}

$\nabla$

Die Entwöhnung von der maschinellen Beatmung ist eine alltägliche Herausforderung für Patienten und medizinisches Personal auf jeder Intensivstation. Gerade bei Patienten, die $>24 \mathrm{~h}$ maschinell beatmet werden müssen, ist eine präzise Abklärung der Ursachen erforderlich. Die Ursachen für die Abhängigkeit von der maschinellen Beatmung oder das schwierige Entwöhnen sind komplex und können sowohl intra- als auch extrapulmonal $\operatorname{sein}($ z.B. $\odot$ Tab. 1).
Die Herz-Kreislauf-Funktion spielt eine große Rolle bei der Entwöhnung von der Beatmung (engl. Weaning). Daher basiert die Entscheidung zur Einleitung der Entwöhnung (readiness to wean), zum Abbruch des Spontanatmungsversuchs (spontaneous breathing trial failure) oder zur Re-Intubation (extubation failure) wesentlich auf kardiovaskulären Parametern, wie z.B. [1,2]:

- readiness to wean: stabile kardiozirkulatorische Situation (d. h. Herzfrequenz $\leq 140 / \mathrm{min}$, systolischer Blutdruck 90 - $160 \mathrm{~mm} \mathrm{Hg}$, keine oder gering dosierte Vasopressoren) 


\begin{tabular}{|c|c|}
\hline \multicolumn{2}{|c|}{ Dysfunktion der Atmungsmuskulatur und Atemmechanik } \\
\hline \multirow{3}{*}{$\begin{array}{l}\text { Erschöpfung der Atmungs- } \\
\text { muskulatur }\end{array}$} & COPD \\
\hline & Adipositas permagna \\
\hline & Anämie \\
\hline \multirow[t]{3}{*}{ Schwäche der Atemmuskulatur } & Neuromuskuläre Erkrankungen \\
\hline & Steroidmyopathie \\
\hline & Malnutrition \\
\hline \multicolumn{2}{|c|}{ Weitere pulmonale und extrapulmonale Ursachen } \\
\hline \multicolumn{2}{|c|}{$\begin{array}{l}\text { Nosokomiale Infekte und } \\
\text { rezidivierende Aspirationen }\end{array}$} \\
\hline \multirow[t]{3}{*}{ Komorbidität } & Kardiale Dysfunktion \\
\hline & Mangelernährung \\
\hline & $\begin{array}{l}\text { Delirium und } \\
\text { neurophysiologische Defizite }\end{array}$ \\
\hline
\end{tabular}

- spontaneous breathing trial failure: Herzfrequenz >140/min, systolischer Blutdruck $>180$ mmHg oder Anstieg um > 20\%, systolischer Blutdruck $<90 \mathrm{mmHg}$, kardiale Arrythmien.

- extubation failure: Herzfrequenz > 140/min oder anhaltender Anstieg $>20 \%$.

Unter maschineller Beatmung, beim Weaning und nach Extubation kommt es zu signifikanten Veränderungen der hämodynamischen Verhältnisse [3]. In Studien wurden vermehrt myokardiale Ischämien und Herzinsuffizienz beim Weaning von Risikopatienten beobachtet [4-7].

Folgende Pathomechanismen können bei Patienten mit eingeschränkter kardiozirkulatorischer Funktion den Übergang von maschineller Beatmung zur Spontanatmung erschweren:

- Erhöhter metabolischer Bedarf beim Weaning und nach Extubation durch vermehrte Atemmuskelarbeit (oxygen cost of breathing) [8] steigert die Anforderungen an das kardiozirkulatorische System (cardiac workload). Erhöhte Laktat-Serumspiegel bzw. eine erniedrigte gemischtvenöse Sauerstoffsättigung oder pH der Magenmucosa sind als Maß der gestörten Perfusion und Sauerstoffausschöpfung der Peripherie und als Prädiktoren eines Weaningversagens beschrieben [4,9].

- Unter der zusätzlichen kardialen Belastung während des Weanings wurden bei Patienten ohne bislang bekannte Herzerkrankung myokardiale Ischämien ausgelöst, die zu linksventrikulärer Dysfunktion, akutem Lungenödem und zum Scheitern des Weanings führten [6]. Ein besonderer Aspekt ist die psychische Erregung (Agitation), die während des Weanings auftreten und zu Anstieg von Herzfrequenz, Blutdruck, Sympathikotonus, cardiac load und Weaningversagen beitragen kann.

- Durch Umkehr vom positiven intrathorakalen Druck unter Beatmung auf negative intrathorakale Druckschwankungen bei der oftmals vertieften Spontanatmung wird der venöse Rückstrom gesteigert (ventrikuläre Vorlast) und zusätzlich

- Zunahme des transmuralen Druckgradienten im Ventrikel (Nachlaststeigerung!). In dieser Situation wurde eine linksventrikuläre Dysfunktion (d.h. Anstieg des pulmonalkapillären Verschlussdrucks von 8 auf $25 \mathrm{mmHg}$ ) während eines erfolglosen Weaningversuches bei 15 Patienten mit COPD beobachtet. Nach forcierter Diurese konnten 9 Patienten erfolgreich entwöhnt werden [5].

- Dynamische Überblähung (intrinsischer oder autopositiver endexspiratorischer Druck, PEEP) mit Steigerung des pulmonalvaskulären Widerstandes und Abfall des venösen Rückstroms und des Herzzeitvolumens.
- Unbekannte Begleiterkrankung: Als Beispiele sei hier einerseits auf die sonografische Abklärung eines unilateral gelähmten Hemidiaphragmas nach (kardio)-chirurgischem Eingriff hingewiesen, die das Weaning erheblich erschweren kann. Andererseits kann die linksventrikuläre Ejektionsfraktion im Rahmen einer prolongierten Sepsis bei 40 - 50\% der Patienten erheblich reduziert sein (sog. septic cardiomyopathy) [10]. Dieser zytokin- und NO-vermittelten reversiblen biventrikulären Dysfunktion liegen multiple Pathomechanismen, einschließlich Downregulation der Betarezeptoren, reduzierte Kalziumfreisetzung aus dem sakroplasmatischen Retikulum und gestörte elektromechanische Kopplung auf Niveau der Myofibrillen zugrunde [10]. Eine konsequente Therapie der auslösenden Entzündung bzw. Infektion ist nicht nur für die Restitution der kardialen Funktion unumgänglich, sondern kann angesichts der nachfolgend dargestellten kardio-pulmonalen Interaktionen über den Weaningerfolg entscheiden.

\section{Kardiovaskuläre Vorerkrankung des Patienten beachten}

Die pathologische Interaktion von Hämodynamik und Ventilation bei kardialer Dysfunktion ist komplex und wird oft übersehen. Bereits durch Anamnese und Basisdiagnostik identifizieren die entscheidenden kardio-zirkulatorischen Faktoren für ein erfolgreiches Weaning und ermöglichen frühzeitiges Eingreifen. Häufig sind bei Patienten kardiale Vorerkrankungen bekannt, z. B. systolische bzw. diastolische ventrikuläre Dysfunktion (oft nur latent), ischämische Kardiomyopathie, hypertrophische obstruktive Kardiomyopathie und angeborene oder erworbene Vitien $[11,12]$. Sowohl Patienten mit Links- als auch mit Rechtsherzinsuffizienz zeigen oftmals eine Schwäche der Atemmuskulatur $[46,47]$, die das Weaning zusätzlich erschweren kann. In einer multizentrischen Feldstudie mit 1419 Patienten, die sich infolge Langzeitbeatmung in Weaningzentren befanden, wiesen 54\% der Patienten eine signifikante kardiale Begleiterkrankung auf [13].

Schwieriger sind die Patienten zu identifizieren, deren myokardiale Dysfunktion erst durch die kardiale Belastung beim Weaning manifest wird. Denn der Übergang von nicht-invasiver oder invasiver maschineller Beatmung zur Spontanatmung kann mit erheblichen hämodynamischen Veränderungen verbunden sein. Die Wiederherstellung der negativen intrathorakalen Druckverhältnisse (d.h. Feuchte Nase, Extubation) erhöht die ventrikuläre Vor- bzw. Nachlast und letztendlich den myokardialen Sauerstoffverbrauch. D.h. eine latente oder bisher unbekannte kardiale Erkrankung kann beim Beginn des Weanings erstmals klinisch manifest werden $[3,5]$.

Diese Beobachtung wird durch die positiven hämodynamischen und prognostischen Effekte der nicht-invasiven Applikation von continous positive airway pressure (CPAP) bei kardiogenem Lungenödem unterstützt [14-16]. Konsequenterweise wird daher die Applikation von CPAP bei Patienten mit akutem kardiogenem Lungenödem eindeutig empfohlen [17].

Beim Weaning spielt die häufig vorkommende Komorbidität von kardiovaskulären Erkrankungen (z.B. koronare Herzerkrankung) und COPD eine besondere Rolle. Mehrere große Kohortenstudien haben den Zusammenhang zwischen reduzierter $\mathrm{FEV}_{1}$ und erhöhtem kardiovaskulärem Mortalitätsrisiko gezeigt [18]. Gerade bei Patienten mit schwerer COPD ist die Atemmuskulatur durch 
die resistive Komponente besonders beansprucht und mitunter erschöpft. Diese vermehrte Atemarbeit erhöht den Sauerstoffbedarf und damit den erforderlichen Anteil am Herzzeitvolumen (oxygen cost of breathing) [8]. Eine solche Steigerung des Herzzeitvolumens im Weaning ist gerade für Patienten mit kardialer Vorerkrankung besonders kritisch (siehe unten).

\section{Erhöhter metabolischer Bedarf der Atmungsmuskulatur beim Weaning}

Ein wesentlicher Unterschied zwischen maschineller Beatmung mit passiver Expansion der Lunge durch positiven Inspirationsdruck und der aktiven Inspiration durch vermehrte Atemmuskelarbeit nach Extubation ist der höhere Sauerstoffverbrauch, der eine Steigerung des Herzzeitvolumens erfordert (siehe $\bullet$ Abb. 1) [19].

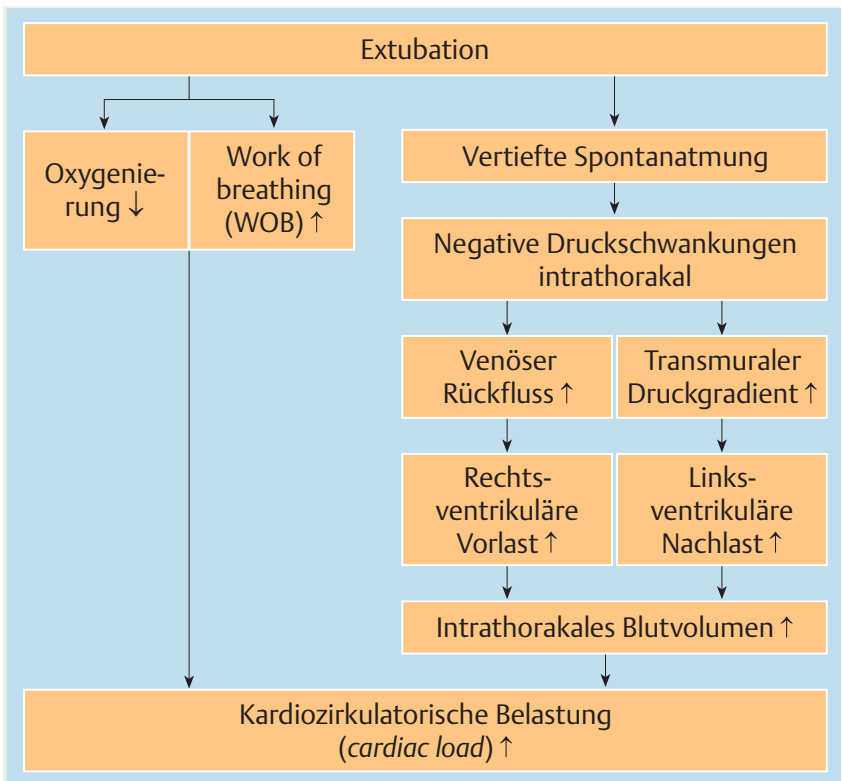

Abb. 1 Effekte der Extubation auf das kardiozirkulatorische System (modifiziert nach [22])

Angesichts des erhöhten Sauerstoffverbrauchs durch vermehrten Einsatz der Atemmuskulatur unter Spontanatmung wurde das Monitoring der Gewebeoxygenierung beim Weaning durchgeführt. Bei Patienten mit erfolgreichem Weaning war während des Spontanatmungsversuchs die Steigerung des Sauerstofftransportes für den gesteigerten Verbrauch ausreichend, sodass die gemischtvenöse Sauerstoffsättigung $\left(\mathrm{SvO}_{2}\right)$ unverändert blieb [4]. Andererseits fiel bei Patienten mit erfolglosem Weaning die $\mathrm{SvO}_{2}$ ab als Hinweis auf inadäquate Steigerung des Sauerstoffangebots, insbesondere des Herzzeitvolumens, d.h. der kardialen Leistungsreserve [4].

Die Rolle der Gewebeoxygenierung zum Monitoring und als Prädiktor eines erfolgreichen Weanings wurde in jüngeren Studien bestätigt $[20,21]$. Allerdings waren die beobachteten Reaktionen während des Spontanatmungsversuchs inhomogen [20]. Ein Abfall der $\mathrm{SvO}_{2}$ wurde nicht bei allen Patienten mit erfolglosem Weaning beobachtet. Eine mögliche Erklärung wäre eine Depression des Atemzentrums bzw. eine Sepsis, sodass kein gesteigerter Sauerstoffverbrauch bei diesen Patienten vorlag [22].
Die Spontanatmung (Feuchte Nase, Extubation) ist bei Patienten mit Linksherzinsuffizienz oftmals gesteigert. Dies erhöht den Sauerstoffverbrauch der Atemmuskulatur, vor allem des Diaphragmas, und kann im Sinne eines steal effects zunehmende Anteile des Herzzeitvolumens beanspruchen und zu einer kritischen myokardialen Ischämie beitragen [19].

\section{Effekte von Weaning und Extubation auf das kardiozirkulatorische System \\ $\nabla$}

Der signifikante Einfluss von Atmung und Beatmung auf die kardiovaskuläre Funktion wird von komplexen, teilweise gegensinnigen Mechanismen vermittelt. Diese Prozesse reflektieren die Interaktion zwischen myokardialer Reserve, ventrikulärer Pumpfunktion, zirkulierendem Blutvolumen, Perfusionsverteilung, Status des autonomen Nervensystems, endokrinologischen Funktionen, Lungenvolumen, intrathorakalem Druck und die Umgebungsdruckverhältnisse der peripheren Zirkulation [3]. Es liegt auf der Hand, dass die kardiovaskuläre Situation des Patienten seine Reaktion auf eine respiratorische Insuffizienz und die Initiierung der Beatmung und deren Entwöhnung wesentlich beeinflusst.

Der abrupte Wechsel von maschineller Beatmung auf Spontanatmung kann bei Patienten mit vorbestehender kardialer Erkrankung zu einem akuten kardiogenen Lungenödem führen. Bei den zugrundeliegenden Pathomechanismen spielt der Wechsel von positiven zu negativen intrathorakalen Druckverhältnissen eine große Rolle, da zum einen eine Steigerung des venösen Rückstroms (ventrikuläre Vorlast) und zum anderen eine Zunahme des transmuralen Druckgradienten der Ventrikel (Nachlast) resultiert (siehe Abb. 1) [3,23]. Gerade bei Patienten mit vorbestehender linksventrikulärer Dysfunktion kann es durch Zunahme des intrathorakalen Blutvolumens und zum Anstieg des pulmonalen Filtrationsdrucks zu einem akuten Lungenödem nach Extubation kommen.

Mitunter führt die deutliche Vor- und Nachlasterhöhung des rechten Ventrikels nach Extubation zur Verlagerung des interventrikulären Septums zulasten des linken Ventrikels (ventricular interdependence). Dadurch wird die diastolische Füllung des linken Ventrikels gestört und die pulmonalvenöse Druckerhöhung nimmt weiter zu [5].

Das abrupte Beenden der maschinellen Beatmung (z.B. T-Stück oder sog. Feuchte Nase) steigert den Sympathikotonus und die Anforderungen an die Atemmuskulatur (work of breathing) wie oben dargestellt [22].

Eine eingeschränkte Herzfrequenzvariabilität beim Spontanatmungsversuch ist sowohl bei Patienten mit Weaningversagen als auch bei Herzinsuffizienz beschrieben [24].

Durch die vermehrte Atemarbeit und die erforderliche Zunahme des Herzzeitvolumens kann eine myokardiale Ischämie bei Patienten mit koronarer Herzkrankheit erstmals manifest werden. Entsprechend wurde das unerwartete Auftreten von kardiogenem Lungenödem bzw. myokardialer Ischämie beim Weaning mehrfach beschrieben $[6,12,25]$.

Eine myokardiale Ischämie kann sowohl als Folge als auch als Ursache eines erfolglosen Weanings angesehen werden [12]. Latente myokardiale Ischämien fanden sich häufig in einem unselektierten Kollektiv internistischer Intensivpatienten, obwohl nur bei wenigen Patienten eine koronare Herzerkrankung bekannt war [26]. Daher sollten Intensivmediziner durch geeignetes Monitoring (z.B. EKG, Troponin) auf das potenzielle Risiko der Mani- 


\begin{tabular}{|c|c|c|}
\hline Erhöhte Vorlast & $\begin{array}{l}\uparrow \text { venöser Rückstrom } \\
\downarrow \text { linksventrikuläre Compliance }\end{array}$ & $\begin{array}{l}\text { Negativer intrathorakaler Druck } \\
\text { Myokardiale Ischämie } \\
\downarrow \mathrm{PaO}_{2}, \downarrow \mathrm{SaO}_{2} \\
\uparrow \mathrm{LVEDP} \uparrow \text { Herzfrequenz, } \downarrow \text { Koronarblutfluss } \\
\uparrow \text { Katecholamine } \\
\uparrow \text { Herzfrequenz, } \uparrow \text { systolischer Blutdruck } \\
\uparrow \text { work of breathing } \\
\text { Links- und rechtsventrikuläre Dilatation }\end{array}$ \\
\hline Reduzierte Kontraktilität & & Myokardiale Ischämie \\
\hline Steigerung der Nachlast & $\begin{array}{l}\text { Dynamische Überblähung } \\
\text { (intrinsic PEEP) }\end{array}$ & $\begin{array}{l}\uparrow \text { systolischer Blutdruck } \\
\text { Negativer intrathorakaler Druck } \\
\uparrow \text { pulmonalvaskulärer Widerstand }\end{array}$ \\
\hline
\end{tabular}

Tab. 2 Faktoren, die beim Weaning zum Anstieg des pulmonalkapillären Verschlussdrucks und Lungenödem führen können (mod. nach [5]) festation einer bis dahin latenten myokardialen Ischämie durch die gesteigerten Anforderungen beim Weaning achten. Unerkannt kann eine myokardiale Ischämie den Weaningerfolg und das Leben des Patienten gefährden [6,12].

Zur Sicherung der Diagnose eines Weaning-assoziierten kardiogenen Lungenödems ist eine Pulmonalis-Katheterisierung und der Nachweis eines Anstiegs des pulmonalkapillären Verschlussdrucks bei Spontanatmung erforderlich [27]. Der Anstieg des pulmonalkapillären Verschlussdrucks während eines Spontanatmungsversuchs kann allerdings multifaktoriell bedingt sein (siehe Tab. 2). Bisher wurde der Stellenwert der echokardiografischen Druckabschätzung in diesem Zusammenhang nicht untersucht [27].

\section{Atriales und B-typ natriuretisches Peptid}

B-typ natriuretische Peptide (BNP) sind quantitative Marker der Herzinsuffizienz und/oder des kardialen Stresses, die das Ausmaß einer systolischen bzw. diastolischen linksventrikulären Dysfunktion, einer valvulären Dysfunktion oder rechtsventrikulären Dysfunktion widerspiegeln [28]. Obwohl der exakte Mechanismus der BNP-Freisetzung noch ungeklärt ist, scheint der enddiastolische Wandstress im Ventrikel eine Schlüsselrolle zu spielen [28]. Daher bieten sich BNP-Plasmaspiegel dem Intensivmediziner an, die linksventrikuläre Toleranz beim Weaning von der maschinellen Beatmung vorherzusagen.

Die Bestimmung von Plasma B-typ natriuretischem Peptid (BNP) kann beim Weaning als humoraler Marker wichtige Hinweise auf linksventrikuläre Dimension und Funktion liefern [22]. In einer Studie bei 102 Patienten charakterisierten signifikant höhere BNP-Plasmaspiegel vor dem Spontanatmungsversuch die Patienten mit Weaningversagen (siehe $\bullet$ Tab. 3) [29]. Allerdings waren BNP-Plasmaspiegel vor und $1 \mathrm{~h}$ nach dem Spontanatmungsversuch bei Patienten mit erfolgreichem und erfolglosem Weaning gleich. Dies wurde damit erklärt, dass $1 \mathrm{~h}$ angesichts der de novo-Synthese von BNP zu kurz war.

Demgegenüber konnte in einer weiteren Untersuchung gezeigt werden, dass der Anstieg des BNP-Plasmaspiegels als Ausdruck der akuten linksventrikulären Nachlaststeigerung beim Spontanatmungsversuch dem Ausgangswert als Prädiktor des Weaningversagens überlegen war [30].

In der jüngsten Arbeit waren die Ausgangswerte für BNP im Plasma bei den Patienten mit erfolglosem und erfolgreichem Weaning gleich [31]. Aber ein Anstieg des BNP-Plasmaspiegels um $<20 \%$ nach einem Spontanatmungsversuch über $2 \mathrm{~h}$ konnte die Patienten mit erfolgreichem Weaning zuverlässig identifizieren [31].
In einer anderen Studie konnte eine gute Diskriminierung zwischen Patienten mit erfolgreichem Weaning und Patienten mit Weaningversagen aufgrund kardialer Ursache durch Bestimmung des erhöhten Anstiegs von NT-proBNP im Plasma nach dem Spontanatmungsversuch im Vergleich zum Ausgangswert erreicht werden [32].

Es bleibt abzuwarten, ob die Bestimmung von BNP oder NTproBNP hilft, eine bessere Selektion von Patienten und Zeitpunkt für ein erfolgreiches Weaning vornehmen zu können. Weitere Studien sollten klären, ob angesichts der relativ kurzen Zeitintervalle zwischen Bestimmung der Plasmaspiegel vor und nach Spontanatmungsversuch das atriale natriuretische Peptid (ANP) bzw. proANP die Patienten mit hohem kardialen Risiko noch zuverlässiger identifiziert. Denn ANP wird aus atrialen Speichern freigesetzt, während BNP konstitutiv gebildet wird.

Besonders hilfreich für den Intensivmediziner wären kardiozirkulatorische Parameter, die ein Weaningversagen vorhersagen, solange der Patient noch maschinelle Beatmung erhält. Auf den Stellenwert von einem erhöhten BNP-Plasmaspiegel vor dem Spontanatmungsversuch wurde oben bereits eingegangen [29]. Diese Ergebnisse stehen im Einklang mit der Beobachtung, dass eine positive Flüssigkeitsbilanz mit Weaningversagen korreliert [33]. Trotz dieser übereinstimmenden Ergebnisse fehlen bisher Daten, die für den systematischen Einsatz von Diuretika mit dem Ziel der Senkung von BNP-Plasmaspiegeln vor Weaningbeginn zur rascheren Entwöhnung sprechen.

\section{Therapeutische Optionen}

Sobald beim Patienten eine kardiozirkulatorische Ursache für das Weaningversagen identifiziert worden ist, muss die konsequente Therapie der kardialen Grunderkrankung (z.B. kritische Koronarstenose) erfolgen.

Nicht zu unterschätzen ist die konsequente medikamentöse Kontrolle einer arteriellen Hypertonie. Ein systemarterieller Blutdruckanstieg $>180 \mathrm{mmHg}$ bzw. um > 20\% vom Ausgangswert stellt eine rapide Nachlaststeigerung für den linken Ventrikel dar (z.B. durch Agitation) und führt gerade bei Patienten mit linksventrikulärer Dysfunktion häufig zu vorzeitigem Abbruch der Weaningphase bzw. des Spontanatmungsversuchs (siehe - Tab. 3) [1].

Bei arterieller Hypotonie oder inadäquatem Herzzeitvolumen setzt man neben der spezifischen Therapie vor allem Diuretika und positiv inotrope Substanzen mit dem Ziel, eine pulmonalvenöse Stauung und ein Lungenödem zu vermeiden, ein [5].

Zur Effektivität von Katecholaminen oder Phosphodiesterase-Inhibitoren in dieser Situation liegen keine aktuellen Studien vor. 


\begin{tabular}{|c|c|c|c|}
\hline Parameter & & Grenzwert & $\begin{array}{l}\text { Literatur- } \\
\text { hinweis }\end{array}$ \\
\hline \multirow[t]{2}{*}{ Herzfrequenz } & $\begin{array}{l}\text { Während Spontanatmungs- } \\
\text { versuch }\end{array}$ & $<140 \mathrm{~min}^{-1}$ & {$[1,2]$} \\
\hline & $\begin{array}{l}\text { Während Spontanatmungs- } \\
\text { versuch }\end{array}$ & Keine Arrhythmien & {$[1,2]$} \\
\hline Systolischer Blutdruck & $\begin{array}{l}\text { Während Spontanatmungs- } \\
\text { versuch }\end{array}$ & $\begin{array}{l}<180 \mathrm{~mm} \mathrm{Hg} \text { oder Anstieg um } \\
<20 \% \text {; systolischer Blutdruck } \\
>90 \mathrm{mmHg}\end{array}$ & {$[1,2]$} \\
\hline $\begin{array}{l}\text { Pulmonalkapillärer } \\
\text { Verschlussdruck }\end{array}$ & $\begin{array}{l}\text { Während Spontanatmungsver- } \\
\text { such über } 10 \mathrm{~min}\end{array}$ & $<15 \mathrm{mmHg}$ & [5] \\
\hline Flüssigkeitsbilanz & & negativ & [33] \\
\hline \multirow[t]{2}{*}{$\begin{array}{l}\text { B-typ natriuretisches Peptid } \\
\text { (BNP) }\end{array}$} & $\begin{array}{l}\text { Plasmaspiegel vor Spontan- } \\
\text { atmungsversuch }\end{array}$ & $<275 \mathrm{pg} / \mathrm{ml}$ & [29] \\
\hline & $\begin{array}{l}\text { Plasmaspiegel nach Spontan- } \\
\text { atmungsversuch über } 120 \text { min }\end{array}$ & $\begin{array}{l}\text { Anstieg }<20 \% \text { vom Ausgangs- } \\
\text { wert }\end{array}$ & [31] \\
\hline $\mathrm{N}$-terminal proBNP & $\begin{array}{l}\text { Plasmaspiegel nach Spontan- } \\
\text { atmungsversuch }\end{array}$ & Anstieg um $\leq 184,7 \mathrm{pg} / \mathrm{ml}$ & {$[32]$} \\
\hline
\end{tabular}

Tab. 3 Auswahl kardiozirkulatorischer Parameter, die in Studienkollektiven mit erfolgreichem Weaning und Extubation assoziiert waren.
Wenn möglich sollte jedoch im Weaningprozess auf die Katecholamine verzichtet werden, denn Katecholamine, durch deren positive Chrono- und Inotropie der Katecholamine und den resultierenden gesteigerten Sauerstoffbedarf kann es gerade bei Patienten mit kardialen Begleiterkrankungen zur Linksherzinsuffizienz kommen.

Vielmehr konnte in einer kürzlich veröffentlichten prospektiven Beobachtungsstudie bei 12 difficult to wean-Patienten durch die 24h-Gabe des Kalziumsensitizers Levosimendan die reduzierte linksventrikuläre Pumpfunktion verbessert werden und in 7 Fällen erfolgreich extubiert werden [34]. Der Stellenwert von Levosimendan als neue vielversprechende Therapieoption beim schwierigen Weaning sollte durch größere Studien evaluiert werden.

Erwiesen ist der günstige Effekt beim Weaning für die Korrektur einer Anämie mittels Bluttransfusion vor allem bei COPD-Patienten $[35,36]$. Es wird postuliert, dass durch Anheben des Sauerstoffgehaltes im Blut der Ventilationsbedarf, die Atemarbeit und somit der Sauerstoffbedarf der Atemmuskulatur sinken und eine „kardiale Entlastung“ ermöglicht wird.

Insbesondere bei Patienten mit kardiovaskulärer Komorbidität ist die Wahl des richtigen Beatmungsregimes entscheidend. Eine Entlastung der Atemmuskulatur und damit eine maximale Reduktion der Atemarbeit lassen sich nur durch die kontrollierte Beatmung (z.B. BIPAP) erzielen [37]. Bereits das „Antriggern“ des Beatmungsgerätes zeigt eine Beanspruchung vor allem des Zwerchfells an. Die negative Druckschwankung intrathorakal kann die ventrikuläre Nachlast und damit den cardiac load steigern (siehe oben).

Andererseits kann durch konsequente Entlastung einer überlasteten Atempumpe (Indikator: Hyperkapnie an Feuchter Nase oder nach Extubation) mittels kontrollierter Beatmungsmodi eine kardiale Rekompensation gelingen. Dies unterstreicht die enge Beziehung zwischen kardiozirkulatorischer Funktion und Weaningerfolg.

\section{Bedeutung von CPAP bzw. NIV in der Respirator- entwöhnung und Postextubationsphase}

$\nabla$

Es ist erwiesen, dass sich bei COPD die Erfolgsrate der Respiratorentwöhnung durch frühzeitige Extubation und unmittelbar anschließende NIV signifikant verbessern lässt $[38,39]$. Zusätzlich kommt es hierdurch zur Reduktion der Letalitäts- sowie Reintubations- und Tracheotomierate. In der Postextubationsphase kann die NIV zur Prävention, aber auch zur Therapie einer erneuten ARI erfolgreich eingesetzt werden. Vor allem bei Risikopatienten mit COPD und Hypersekretion, die nach Extubation eine hyperkapnische Atmungsinsuffizienz entwickeln, reduziert der frühzeitige Einsatz von NIV die Reintubations- und Letalitätsrate [40-43]. Analog zu den oben ausgeführten Betrachtungen bzgl. Sauerstoffverbrauch der Atmungsmuskulatur kann postuliert werden, dass zumindest bei beatmeten Patienten mit COPD und gleichzeitiger KHK NIV in der Postextubationsphase auch zur Verbesserung der kardialen Funktion beiträgt [44].

Darüber hinaus ist für das kardial bedingte Lungenödem, eine Sonderform der hypoxämischen ARI, der Effekt von Continuous Positive Airway Pressure (CPAP) und NIV laut Metaanalysen als Intervention neben der medikamentösen Standardtherapie belegt [45]. Beim kardialen Lungenödem bewirkt CPAP das Absenken der kardialen Vor- und Nachlast, die Reduktion der Atemarbeit und eine Verbesserung der Koronarperfusion und normalisiert das Ventilations-Perfusions-Verhältnis. In einer aktuellen Publikation hierzu führten CPAP mit 5-15 $\mathrm{cm} \mathrm{H}_{2} \mathrm{O}$ ) bzw. NIV (inspiratorische Druckunterstützung: $8-20 \mathrm{~cm} \mathrm{H}_{2} \mathrm{O}$ ) im Vergleich zur Standardtherapie zwar zur schnelleren Abnahme der Atemnot, Hyperkapnie, Azidose und Herzfrequenz, jedoch zu keiner Beeinflussung der Kurzzeitsterblichkeit[44].

\section{Literatur}

1 Boles JM, Bion J, Connors A et al. Weaning from mechanical ventilation. Eur Respir J 2007; 29: 1033 - 1056

2 MacIntyre NR, Cook DJ, Ely EW et al. Evidence-based guidelines for weaning and discontinuing ventilatory support. Chest 2001; 120: 375S395S

3 Pinsky MR. Cardiovascular issues in respiratory care. Chest 2005; 128 : 592S-597S

4 Jubran A, Mathru M, Dries D et al. Continuous recordings of mixed venous oxygen saturation during weaning from mechanical ventilation and the ramifications thereof. Am J Resp Crit Care Med 1998; 158: $1763-1769$ 
5 Lemaire F, Teboul JL, Cinotti L et al. Acute Left-Ventricular Dysfunction During Unsuccessful Weaning from Mechanical Ventilation. Anesthesiology 1988; 69: 171-179

6 Chatila W, Ani S, Guaglianone D et al. Cardiac ischemia during weaning from mechanical ventilation. Chest 1996; 109: 1577-1583

7 Mohsenifar Z, Hay A, Hay J et al. Gastric Intramural Ph As A Predictor of Success Or Failure in Weaning Patients from Mechanical Ventilation. Ann Int Med 1993; 119: $794-798$

8 Roussos C, Macklem PR. The respiratory muscles. N Engl J Med 1982; 307: $786-797$

9 Mohsenifar Z, Hay A, Hay J et al. Gastric Intramural Ph As A Predictor of Success Or Failure in Weaning Patients from Mechanical Ventilation. Ann Int Med 1993; 119: $794-798$

10 Rudiger A, Singer M. Mechanisms of sepsis-induced cardiac dysfunction. Crit Care Med 2007; 35: 1599-1608

11 Adamopoulos C, Tsagourias M, Arvaniti $K$ et al. Weaning failure from mechanical ventilation due to hypertrophic obstructive cardiomyopathy. Intensive Care Med 2005; 31: 734-737

12 Srivastava S, Chatila W, Amoateng-Adjepong Y, Kanagasegar S, Jacob B, Zarich S, Manthous CA. Myocardial ischemia and weaning failure in patients with coronary artery disease: An update. Crit Care Med 1999; 27: $2109-2112$

13 Scheinhorn DJ, Hassenpflug MS, Votto JJ et al. Ventilator-dependent survivors of catastrophic illness transferred to 23 long-term care hospitals for weaning from prolonged mechanical ventilation. Chest 2007; 131 : $76-84$

14 Lapinsky SE, Mount DB, Mackey D et al. Management of acute respiratory failure due to pulmonary edema with nasal positive pressure support. Chest 1994; 105: 229-231

15 Naughton MT, Rahman MA, Hara $K$ et al. Effect of continuous positive airway pressure on intrathoracic and left ventricular transmural pressures in patients with congestive heart failure. Circulation 1995; 91: $1725-1731$

16 Vital FMR, Saconato H, Ladeira MT et al. Non-invasive positive pressure ventilation (CPAP or bilevel NPPV) for cardiogenic pulmonary edema. Cochrane Database of Systematic Reviews

17 Dickstein K, Cohen SolalA, Filippatos G et al. ESC Guidelines on the Diagnosis and Treatment of Acute Heart Failure. Eur Heart J 2008; 29 : $2388-2442$

18 MacNee W, Maclay J, McAllister D. Cardiovascular injury and repair in chronic obstructive pulmonary disease. Proc Am Thorac Soc 2008; 5 : $824-833$

19 Wise RA, Robotham JL, Summer WR. Effects of spontaneous ventilation on the circulation. Lung 1981; 159: 175-186

20 Zakynthinos S, Routsi C, Vassilakopoulos T et al. Differential cardiovascular responses during weaning failure: effects on tissue oxygenation and lactate. Intensive Care Med 2005; 31: 1634-1642

21 Mohsenifar Z, Hay A, Hay J et al. Gastric Intramural Ph As A Predictor of Success Or Failure in Weaning Patients from Mechanical Ventilation. Ann Int Med 1993; 119: 794 - 798

22 Monnet X, Teboul JL, Richard C. Cardiopulmonary interactions in patients with heart failure. Curr Opin Crit Care 2007; 13: 6-11

23 Richard C, Teboul JL, Archambaud F et al. Left-Ventricular Function During Weaning of Patients with Chronic Obstructive Pulmonary-Disease. Intensive Care Med 1994; 20: 181 - 186

24 Shen $H N$, Lin $L Y$, Chen $K Y$ et al. Changes of heart rate variability during ventilator weaning. Chest 2003; 123: $1222-1228$

25 Hurford WE, Favorite F. Association of Myocardial-Ischemia with Failure to Wean from Mechanical Ventilation. Crit Care Med 1995; 23: $1475-1480$

26 Frazier SK, Brom H, Widener J et al. Prevalence of myocardial ischemia during mechanical ventilation and weaning and its effects on weaning success. Heart \& Lung 2006; 35: 363 - 373
27 Teboul JL, Richard C. How to diagnose weaning-induced pulmonary edema? Intensive Care Med 2006; 32: 938

28 Mueller C, Breidthardt T, Laule-Kilian K et al. The integration of BNP and NT-proBNP into clinical medicine. Swiss Medical Weekly 2007; 137: 4-12

29 Mekontso-Dessap A, De Prost N, Girou E et al. B-type natriuretic peptide and weaning from mechanical ventilation. Intensive Care Med 2006; 32: $1529-1536$

30 Ait-Oufella H, Tharaux PL, Baudel JL et al. Variation in natriuretic peptides and mitral flow indexes during successful ventilatory weaning: a preliminary study. Intensive Care Med 2007; 33: 1183-1186

31 Chien JY, Lin MS, Huang YCT et al. Changes in B-type natriuretic peptide improve weaning outcome predicted by spontaneous breathing trial. Crit Care Med 2008; 36: 1421-1426

32 Grasso S, Leone A, DeMichele $M$ et al. Use of N-terminal pro-brain natriuretic peptide to detect acute cardiac dysfunction during weaning failure in difficult-to-wean patients with chronic obstructive pulmonary disease. Crit Care Med 2007; 35: 96 - 105

33 Upadya A, Tilluckdharry L, Muralidharan $V$ et al. Fluid balance and weaning outcomes. Intensive Care Med 2005; 31: 1643-1647

34 Streba M, Banerjee A, Mudaliar Y. Prospective observational study of levosimendan and weaning of difficult-to-wean ventilator dependent intensive care patients. Crit Care Resusc 2008; 10: 182-186

35 Schönhofer B, Wenzel M, Geibel $M$ et al. Blood transfusion and lung function in chronically anemic patients with severe chronic obstructive pulmonary disease. Crit Care Med 1998; 26: 1824-1828

36 Schönhofer B, Bohrer H, Kohler D. Blood transfusion facilitating difficult weaning from the ventilator. Anaesthesia 1998; 53: $181-184$

37 Schönhofer B, Sonneborn M, Haidl P et al. Nocturnal mechanical ventilation after long-term mechanical ventilation. Med Klin 1996; 91: 27 30

38 Ferrer $M$, Esquinas A, Arancibia $F$ et al. Noninvasive ventilation during persistent weaning failure - A randomized controlled trial. Am J Resp Crit Care Med 2003; 168: 70-76

39 Nava S, Ambrosino N, Clini E et al. Noninvasive mechanical ventilation in the weaning of patients with respiratory failure due to chronic obstructive pulmonary disease - A randomized, controlled trial. Ann Int Med 1998; 128: 721 - 728

40 Carlucci A, Gregoretti C, Squadrone V et al. Preventive use of non-invasive mechanical ventilation to avoid post-extubation respiratory failure: a randomised controlled study. Eur Respir J 2001; 18 Suppl : 306

41 Ferrer $M$, Valencia $M$, Nicolas JM et al. Early noninvasive ventilation averts extubation failure in patients at risk - A randomized trial. Am J Resp Crit Care Med 2006; 173: 164-170

42 Hilbert G, Gruson D, Portel L et al. Noninvasive pressure support ventilation in COPD patients with postextubation hypercapnic respiratory insufficiency. Eur Respir J 1998; 11: 1349-1353

43 Nava S, Gregoretti C, Fanfulla F et al. Noninvasive ventilation to prevent respiratory failure after extubation in high-risk patients. Crit Care Med 2005; 33: $2465-2470$

44 Gray A, Goodacre S, Newby DE et al. Noninvasive ventilation in acute cardiogenic pulmonary edema. N Engl J Med 2008; 359: 142 - 151

45 Winck JC, Azevedo LF, Costa-Pereira A et al. Efficacy and safety of noninvasive ventilation in the treatment of acute cardiogenic pulmonary edema - a systematic review and meta-analysis. Crit Care 2006; 10: R69

46 Meyer FJ, Borst MM, Zugck C et al. Respiratory muscle dysfunction in congestive heart failure: clinical correlation and prognostic significance. Circulation 2001; 103: $2153-2158$

47 Meyer FJ, Lossnitzer D, Kristen AV et al. Respiratory muscle dysfunction in idiopathic pulmonary arterial hypertension. Eur Respir J 2005; 25 : $125-130$ 